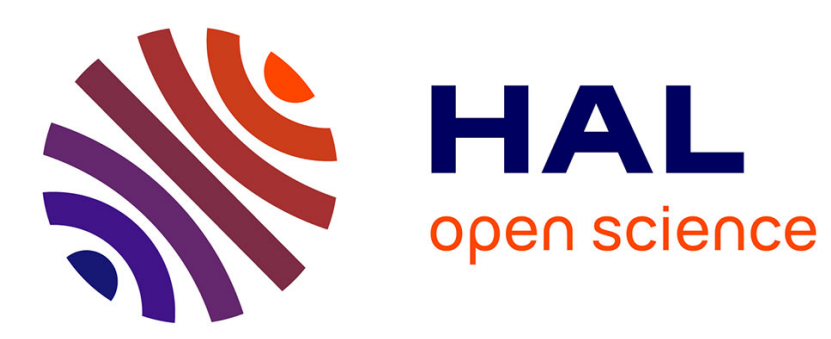

\title{
Remarks on bounded solutions of steady Hamilton-Jacobi equations
}

\author{
Gawtum Namah, Mihai Bostan
}

\section{To cite this version:}

Gawtum Namah, Mihai Bostan. Remarks on bounded solutions of steady Hamilton-Jacobi equations. Comptes rendus de l'Académie des sciences. Série I, Mathématique, 2009, 15-16 (347), pp.873-878. 10.1016/j.crma.2009.06.004 . hal-00476301

\section{HAL Id: hal-00476301 https://hal.science/hal-00476301}

Submitted on 6 May 2010

HAL is a multi-disciplinary open access archive for the deposit and dissemination of scientific research documents, whether they are published or not. The documents may come from teaching and research institutions in France or abroad, or from public or private research centers.
L'archive ouverte pluridisciplinaire HAL, est destinée au dépôt et à la diffusion de documents scientifiques de niveau recherche, publiés ou non, émanant des établissements d'enseignement et de recherche français ou étrangers, des laboratoires publics ou privés. 


\title{
Remarks on bounded solutions of steady Hamilton-Jacobi equations
}

\author{
Quelques remarques sur les solutions bornées des équations \\ stationnaires d'Hamilton-Jacobi \\ Mihaï BOSTAN ${ }^{a}$ Gawtum NAMAH ${ }^{\mathrm{a}}$ \\ a Laboratoire de Mathématiques de Besançon, UMR CNRS 6623, Université de Franche-Comté, 16 route de Gray \\ F-25030 Besançon Cedex, tél : 03.81.66.63.38, fax : 03.81.66.66.23
}

\begin{abstract}
Résumé
Dans cette note on s'intéresse à l'équation $H(D u)=H(0), x \in \mathbb{R}^{N}$ et plus précisément à la question suivante : dans quels cas les fonctions constantes sont-elles les seules solutions bornées de cette équation? On démontre que tel est le cas sous des hypothèses de stricte convexité et coercivité en dimension $N$ quelconque. La preuve fait appel à la formule de Hopf-Lax. En une dimension d'espace on propose un résultat pour des hamiltoniens seulement faiblement coercifs moyennant une condition supplémentaire. Dans la dernière partie on utilise ces résultats pour identifier les limites asymptotiques en temps long des solutions des problèmes de Cauchy.
\end{abstract}

\section{Abstract}

We study here the equation $H(D u)=H(0), x \in \mathbb{R}^{N}$. More precisely we investigate under which hypotheses the constant functions are the only bounded solutions. We start by proving that this is the case under strict convexity and coercivity conditions. We then give a result in one space dimension for hamiltonians which are not necessarily convex. These results apply when studying the long time behaviour of solutions for time-dependent Hamilton-Jacobi equations.

\section{Version Française abrégée}

Soit $H: \mathbb{R}^{N} \rightarrow \mathbb{R}$ une fonction vérifiant

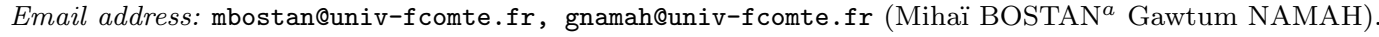




$$
\lim _{|p| \rightarrow+\infty} \frac{H(p)}{|p|}=+\infty .
$$

On montre que les seules solutions (au sens de viscosité) bornées de l'équation stationnaire d'HamiltonJacobi $H(D u)=H(0), x \in \mathbb{R}^{N}$ sont données par les fonctions constantes. Sans perte de généralité on peut supposer que $H(0)=0$. La preuve utilise la formule de représentation de Hopf-Lax [4] pp. 560 . Il est bien connu que si l'hamiltonien $H$ vérifie (1), (2), alors toute solution au sens de viscosité de $H(D u)=0, x \in \mathbb{R}^{N}$ vérifie

$$
u(x)=\inf _{y \in \mathbb{R}^{N}}\left\{u(y)+t L\left(\frac{x-y}{t}\right)\right\}
$$

où $L: \mathbb{R}^{N} \rightarrow \mathbb{R}$ est la fonction conjuguée à $H$ par dualité convexe

$$
L(q)=\sup _{p \in \mathbb{R}^{N}}\{q \cdot p-H(p)\}, \quad q \in \mathbb{R}^{N} .
$$

En une dimension d'espace il est possible d'étudier une classe plus large d'hamiltoniens, pas nécessairement convexes. On considère $H: \mathbb{R} \rightarrow \mathbb{R}$ seulement faiblement coercif i.e.,

$$
\lim _{|p| \rightarrow+\infty} H(p)=+\infty
$$

et vérifiant

$$
0 \notin \overline{H^{-1}(H(0)) \backslash\{0\}} .
$$

En utilisant les notions de sous/sur-différentiel (la formule de Hopf-Lax n'étant plus valide, car $H$ n'est plus supposé convexe) on montre que les seules solutions (au sens de viscosité) bornées de

$$
H\left(u_{x}\right)=H(0), \quad x \in \mathbb{R}
$$

sont les fonctions constantes. Ces résultats s'appliquent lorsqu'on souhaite étudier le comportement en temps long d'un problème d'évolution avec condition initiale

$$
\left\{\begin{array}{l}
\left.\partial_{t} u+H\left(\partial_{x} u\right)=0, \quad(x, t) \in \mathbb{R} \times\right] 0,+\infty[ \\
u(x, 0)=u_{0}(x), \quad x \in \mathbb{R}
\end{array}\right.
$$

\section{Bounded stationary solutions}

The subject matter of this note concerns the stationary equation

$$
H(D u)=H(0), \quad x \in \mathbb{R}^{N}
$$

where $H: \mathbb{R}^{N} \rightarrow \mathbb{R}$ is a continuous function. First we investigate the case of convex hamiltonians.

Proposition 1.1 Let $H=H(p): \mathbb{R}^{N} \rightarrow \mathbb{R}$ satisfying (1), (2) such that its conjugate function is $C^{1}$ and strictly convex in a neighbourhood of its minimum point. Then the constants are the only bounded solutions of (9) (in viscosity sense).

We appeal here to Hopf-Lax representation formula [4] pp. 560. Before detailing the proof let us recall the following standard results concerning the conjugate (by convex duality) function $L$ associated to the hamiltonian $H$. We have (see [4] pp. 122)

Proposition 1.2 (Convex duality of Hamiltonian and Lagrangian) Assume that $H$ satisfies (1), (2). Then the mapping $L$ is convex and satisfies $\lim _{|q| \rightarrow+\infty} L(q) /|q|=+\infty$. Furthemore, the conjugate function associated to $L$ coincides with $H$. 
Notice that (2) guarantees that the supremum in (4) is attained for any $q \in \mathbb{R}^{N}$ and that the mapping $L$ is locally Lipschitz. We check easily that $L(q) \geq-H(0), q \in \mathbb{R}^{N}$. If $H$ is strictly convex, the dual function $L$ enjoys other interesting properties, see for e.g. [3]. Indeed, for any $q \in \mathbb{R}^{N}$, there is a unique $p \in \mathbb{R}^{N}$ such that $\partial H(p) \ni q$ and we have for any element in $\partial H(p)$

$$
L(\partial H(p))=\partial H(p) \cdot p-H(p), \quad p \in \mathbb{R}^{N} .
$$

We also mention that the strict convexity of $H$ ensures that $L$ is $C^{1}$ function and $D L(\partial H(p))=p, p \in \mathbb{R}^{N}$.

Remark 1 Since $H$ coincides with the conjugate function of L, observe that, under the hypotheses of Proposition 1.1, necessarily $H$ is $C^{1}$ around 0 . We also mention that the strict convexity of $H$ around 0 would guarantee that $L$ is $C^{1}$ around its minimum point.

Proof (of Proposition 1.1) Without loss of generality we can assume that $H(0)=0$. First notice that by the coercivity condition (2) any bounded solution is in fact Lipschitz continuous so that it is a.e. differentiable. Therefore it suffices to show that $D u=0$ a.e. to conclude that $u$ is a constant function. For this sake we are going to use the representation Hopf-Lax formula (3) for convex coercive hamiltonians. Let $x_{0}$ be a differentiability point of $u$. We want to show that $D u\left(x_{0}\right)=0$. Let $y_{0}^{t}=y_{0}^{t}\left(x_{0}, t\right)$ be a minimum point in (3) i.e.,

Since $u$ is bounded we have

$$
u\left(x_{0}\right)=u\left(y_{0}^{t}\right)+t L\left(z_{0}^{t}\right), \quad z_{0}^{t}=\frac{x_{0}-y_{0}^{t}}{t} .
$$

$$
L\left(z_{0}^{t}\right) \leq \frac{2\|u\|_{L^{\infty}}}{t}, t>0 .
$$

The boundedness of $L$ together with its coercivity lead then to the boundedness of $\left(z_{0}^{t}\right)_{t>0}$. Thus there is a sequence $\left(t_{k}\right)_{k}$ diverging towards $+\infty$ such that the sequence $\left(z_{0}^{t_{k}}\right)_{k}$ converges to some limit $z_{\infty}$. From (12) we deduce that $L\left(z_{\infty}\right) \leq 0$. As $L(q) \geq-H(0)=0$ for any $q \in \mathbb{R}^{N}$, we conclude that $L\left(z_{\infty}\right)=0$ saying that $z_{\infty}$ is the (unique) minimum point for $L, D L\left(z_{\infty}\right)=0$ and therefore

$$
\lim _{k \rightarrow+\infty} D L\left(z_{0}^{t_{k}}\right)=D L\left(z_{\infty}\right)=0 \text {. }
$$

The idea is to differentiate (11) with respect to $x_{0}$ for any fixed $k$ and then to let $k \rightarrow+\infty$. At least formally one gets

$$
D u\left(x_{0}\right)=D L\left(z_{0}^{t_{k}}\right) \rightarrow D L\left(z_{\infty}\right)=0, \text { as } k \rightarrow+\infty .
$$

The rigorous justification of the previous statement follows by estimating the Lipschitz constant of $u$ around $x_{0}$ for any fixed $t_{k}$ and taking the limit as $k \rightarrow+\infty$. The crucial hypotheses allowing this are the strict convexity and $C^{1}$ regularity of $L$ around $z_{\infty}$. Indeed, let $x \in B\left(x_{0}, 1\right)$ and $y^{t_{k}}$ be a minimum point in (3) i.e.,

$$
u(x)=u\left(y^{t_{k}}\right)+t_{k} L\left(z^{t_{k}}\right), \quad z^{t_{k}}=\frac{x-y^{t_{k}}}{t_{k}} .
$$

Combining (11), (14) yields

$$
\left|L\left(z^{t_{k}}\right)-L\left(z_{\infty}\right)\right| \leq \frac{4\|u\|_{L^{\infty}}}{t_{k}}+\left|L\left(z_{0}^{t_{k}}\right)-L\left(z_{\infty}\right)\right| \rightarrow 0 \text { as } k \rightarrow+\infty .
$$

By the strict convexity of $L$ around its minimum point $z_{\infty}$ one gets

$$
\max \left\{\left|z_{0}^{t_{k}}-z_{\infty}\right|,\left|z^{t_{k}}-z_{\infty}\right|\right\} \leq r\left(1 / t^{k}\right) .
$$

Here the notation $r: \mathbb{R}_{+}^{\star} \rightarrow \mathbb{R}$ stands for various functions such that $\lim _{s}{ }_{0} r(s)=0$. Obviously we have

$$
\left|\frac{x_{0}-y^{t_{k}}}{t_{k}}-z_{\infty}\right| \leq \frac{\left|x_{0}-x\right|}{t_{k}}+\left|z^{t_{k}}-z_{\infty}\right| \leq r\left(1 / t_{k}\right) .
$$


We can write for any $x \in B\left(x_{0}, 1\right)$

$$
\begin{aligned}
u(x)-u\left(x_{0}\right) & =u\left(y^{t_{k}}\right)+t_{k} L\left(\frac{x_{0}-y^{t_{k}}}{t_{k}}\right)-u\left(x_{0}\right)+t_{k}\left(L\left(\frac{x-y^{t_{k}}}{t_{k}}\right)-L\left(\frac{x_{0}-y^{t_{k}}}{t_{k}}\right)\right) \\
& \geq-\Lambda_{k}(L)\left|x-x_{0}\right|
\end{aligned}
$$

where $\Lambda_{k}(L)=\sup \left\{\frac{\left|L(z)-L\left(z^{\prime}\right)\right|}{\left|z-z^{\prime}\right|}: z \neq z^{\prime}, z, z^{\prime} \in B\left(z_{\infty}, r\left(1 / t_{k}\right)\right)\right\}$. Similarly one gets $u\left(x_{0}\right)-u(x) \geq$ $-\Lambda_{k}(L)\left|x-x_{0}\right|$ and thus we deduce that $\left|D u\left(x_{0}\right)\right| \leq \Lambda_{k}(L)$ for any $k$. Finally, letting $k \rightarrow+\infty$ and by taking into account that $L$ is $C^{1}$ around $z_{\infty}$ yield

$$
\left|D u\left(x_{0}\right)\right| \leq \lim _{k \rightarrow+\infty} \Lambda_{k}(L)=\left|D L\left(z_{\infty}\right)\right|=0 .
$$

We now propose a result in one dimension concerning the possible bounded solutions of (7) when $H$ is not necessarily convex, more precisely $H: \mathbb{R} \rightarrow \mathbb{R}$ is continuous and satisfies the coercivity condition (5). For this sake consider the set

$$
I_{H}=\{p \in \mathbb{R}: H(p)=H(0)\} .
$$

Notice that $I_{H}$ is nonempty $\left(0 \in I_{H}\right)$ and closed. We then have

Proposition 1.3 Let $H=H(p)$ satisfy (5) such that $0 \notin \overline{I_{H}-\{0\}}$. Then the constants are the only bounded solutions of (7).

Let us point out that strictly convex hamiltonians satisfy $0 \notin \overline{I_{H}-\{0\}}$. Notice also that just convex is not sufficient as is shown by the following example

$$
H(p)= \begin{cases}-p-1, & p \in]-\infty,-1[, \\ 0, & p \in[-1,1], \\ p-1, & p \in] 1, \infty[,\end{cases}
$$

where possible solutions are (up to additive constants) any function $v$ such that $\left|v^{\prime}(x)\right| \leq 1, x \in$ $\mathbb{R}$. Here we have $\overline{I_{H}-\{0\}}=[-1,1] \ni 0$ and as we see solutions other than constants exist (for e.g. $u(x)=\sin x, x \in \mathbb{R})$. The proof of the above proposition relies on the notions of subdifferential and superdifferential for continuous functions $v \in C(\mathbb{R})$

$$
\begin{aligned}
& D^{-} v(x)=\left\{p \in \mathbb{R}: \liminf _{y \rightarrow x} \frac{v(y)-v(x)-p(y-x)}{|y-x|} \geq 0\right\}, \\
& D^{+} v(x)=\left\{p \in \mathbb{R}: \limsup _{y \rightarrow x} \frac{v(y)-v(x)-p(y-x)}{|y-x|} \leq 0\right\} .
\end{aligned}
$$

We use the following easy lemma

Lemma 1.4 Let $v \in C(\mathbb{R})$ be a continuous function and $x_{1}<x_{2}$ two differentiability points for $v$ such that $v^{\prime}\left(x_{1}\right) \neq v^{\prime}\left(x_{2}\right)$.

(i) If $v^{\prime}\left(x_{1}\right)<k<v^{\prime}\left(x_{2}\right)$ there is $\left.x_{3} \in\right] x_{1}, x_{2}\left[\right.$ such that $D^{-} v\left(x_{3}\right) \ni k$.

(ii) If $v^{\prime}\left(x_{1}\right)>k>v^{\prime}\left(x_{2}\right)$ there is $\left.x_{4} \in\right] x_{1}, x_{2}\left[\right.$ such that $D^{+} v\left(x_{4}\right) \ni k$.

Proof Without loss of generality we can assume $k=0$ (replace the function $v(x)$ by $v(x)-k x, x \in \mathbb{R}$ ).

(i) We assume that $v^{\prime}\left(x_{1}\right)<0<v^{\prime}\left(x_{2}\right)$. Consider $x_{3} \in\left[x_{1}, x_{2}\right]$ such that $v\left(x_{3}\right)=\min \{v(x): x \in$ $\left.\left[x_{1}, x_{2}\right]\right\}$. Obviously we have $x_{3} \neq x_{1}, x_{3} \neq x_{2}$ and therefore

$$
\liminf _{y \rightarrow x_{3}} \frac{v(y)-v\left(x_{3}\right)}{\left|y-x_{3}\right|} \geq 0
$$


saying that $0 \in D^{-} v\left(x_{3}\right)$.

(ii) In the case $v^{\prime}\left(x_{1}\right)>0>v^{\prime}\left(x_{2}\right)$ take $x_{4} \in\left[x_{1}, x_{2}\right]$ such that $v\left(x_{4}\right)=\max \left\{v(x): x \in\left[x_{1}, x_{2}\right]\right\}$. We easily check that $0 \in D^{+} v\left(x_{4}\right)$.

Now we are ready to prove Proposition 1.3.

Proof (of Proposition 1.3) Let $u$ be a bounded solution of (7). Since the hamiltonian satisfies the coercivity condition, $u$ is a Lipschitz function and therefore it is differentiable a.e. on $\mathbb{R}$. We show that the first derivative of $u$ has constant sign. More precisely we prove that if there is $x_{1} \in \mathbb{R}$ such that $u^{\prime}\left(x_{1}\right) \neq 0$ then $u^{\prime}(x) u^{\prime}\left(x_{1}\right)>0$, for a.a. $x \in \mathbb{R}$. To fix the ideas assume that there is $x_{1} \in \mathbb{R}$ such that $u^{\prime}\left(x_{1}\right)<0$, the other case following in similar way. Suppose that there is $x_{2}$ such that $u^{\prime}\left(x_{2}\right)>0$ and let us search for a contradiction.

Case 1. Consider that $x_{1}<x_{2}$. By Lemma 1.4 we know that for any $\left.p \in\right] u^{\prime}\left(x_{1}\right), u^{\prime}\left(x_{2}\right)$ [ there is $\left.x_{p} \in\right] x_{1}, x_{2}$ [ such that $D^{-} u\left(x_{p}\right) \ni p$. Since $u$ is in particular a supersolution of (7) we deduce that

$$
H(p) \geq H(0), \forall p \in] u^{\prime}\left(x_{1}\right), u^{\prime}\left(x_{2}\right)[.
$$

We use now the hypothesis $0 \notin \overline{I_{H}-\{0\}}$ which is equivalent to

$$
\exists \varepsilon>0: H(p) \neq H(0), \forall p \in]-\varepsilon, 0[\cup] 0, \varepsilon[\text {. }
$$

Obviously since $u^{\prime}\left(x_{1}\right)<0, u^{\prime}\left(x_{2}\right)>0, H\left(u^{\prime}\left(x_{1}\right)\right)=H\left(u^{\prime}\left(x_{2}\right)\right)=H(0)$ we obtain $u^{\prime}\left(x_{1}\right) \leq-\varepsilon<\varepsilon \leq$ $u^{\prime}\left(x_{2}\right)$. It remains to notice that $u^{\prime}\left(x_{3}\right) \geq \varepsilon$ for a.a. $x_{3}>x_{2}$. Indeed if there is $x_{3}>x_{2}$ such that $u^{\prime}\left(x_{3}\right)<\varepsilon \leq u^{\prime}\left(x_{2}\right)$ by Lemma 1.4 we know that for any $\left.p \in\right] u^{\prime}\left(x_{3}\right), u^{\prime}\left(x_{2}\right)\left[\right.$ there is $\left.y_{p} \in\right] x_{2}, x_{3}[$ such that $D^{+} u\left(y_{p}\right) \ni p$. Since $u$ is in particular a subsolution of (7) we deduce that

$$
H(p) \leq H(0), \quad p \in] u^{\prime}\left(x_{3}\right), u^{\prime}\left(x_{2}\right)[.
$$

Combining (16), (18) we obtain $H(p)=H(0)$ for any

$$
p \in] \max \left\{u^{\prime}\left(x_{1}\right), u^{\prime}\left(x_{3}\right)\right\}, u^{\prime}\left(x_{2}\right)[,
$$

which is not possible in view of (17) and of the inequalities $\max \left\{u^{\prime}\left(x_{1}\right), u^{\prime}\left(x_{3}\right)\right\}<\varepsilon, u^{\prime}\left(x_{2}\right) \geq \varepsilon$. Thus the inequality $u^{\prime}\left(x_{3}\right) \geq \varepsilon$ holds for a.a. $x_{3}>x_{2}$ leading to a contradiction since in this case $u\left(x_{3}\right)$ becomes unbounded when $x_{3}$ goes to $+\infty$

$$
u\left(x_{3}\right) \geq u\left(x_{2}\right)+\varepsilon\left(x_{3}-x_{2}\right), \forall x_{3}>x_{2} .
$$

Case 2. Consider now that $x_{1}>x_{2}$. Combining Lemma 1.4 and the fact that $u$ is a subsolution for $(7)$ yields

$$
H(p) \leq H(0), \forall p \in] u^{\prime}\left(x_{1}\right), u^{\prime}\left(x_{2}\right)[.
$$

Assume that there is $x_{3}<x_{2}$ such that $u^{\prime}\left(x_{3}\right)<\varepsilon$. By Lemma 1.4 combined with the fact that $u$ is a supersolution for (7) we obtain

$$
H(p) \geq H(0), \quad p \in] u^{\prime}\left(x_{3}\right), u^{\prime}\left(x_{2}\right)[,
$$

and therefore

$$
H(p)=H(0), \quad p \in] \max \left\{u^{\prime}\left(x_{1}\right), u^{\prime}\left(x_{3}\right)\right\}, u^{\prime}\left(x_{2}\right)[,
$$

which is not possible in view of (17). Thus $u^{\prime}\left(x_{3}\right) \geq \varepsilon$ for a.a. $x_{3}<x_{2}$ leading to a contradiction since in this case $u\left(x_{3}\right)$ becomes unbounded when $x_{3}$ goes to $-\infty$

$$
u\left(x_{3}\right) \leq u\left(x_{2}\right)-\varepsilon\left(x_{2}-x_{3}\right), \forall x_{3}<x_{2} .
$$

Once we have proved that $u^{\prime}$ has constant sign it is easily seen that every bounded solution for (7) is constant. Indeed if there is $x_{1} \in \mathbb{R}$ such that $u^{\prime}\left(x_{1}\right)>0$ we know that $u^{\prime}(x)>0$ for a.a. $x \in \mathbb{R}$. Since $H\left(u^{\prime}(x)\right)=H(0)$ for a.a. $x \in \mathbb{R}$ we deduce by $(17)$ that $u^{\prime}(x) \geq \varepsilon$ for a.a $x \in \mathbb{R}$ and therefore $u$ does not remain bounded. If there is $x_{2} \in \mathbb{R}$ such that $u^{\prime}\left(x_{2}\right)<0$ we obtain a contradiction in a similar manner. Thus $u^{\prime}(x)=0$ for a.a. $x \in \mathbb{R}$ saying that $u$ is constant. 


\section{An application : explicit limiting solutions}

The previous results enable us in certain cases to give explicitly the limiting solutions of initial value problems. We give here two examples.

Example 1 Consider the initial value problem

$$
\left\{\begin{array}{l}
\left.\partial_{t} u+H\left(\partial_{x} u\right)=0, \quad(x, t) \in \mathbb{R} \times\right] 0,+\infty[, \\
u(x, 0)=u_{0}(x), \quad x \in \mathbb{R}
\end{array}\right.
$$

such that

Then we have the following

$$
H(0)=0, \quad 0 \notin \overline{I_{H}-\{0\}} \text {. }
$$

Proposition 2.1 Let $H=H(p)$ satisfy (5), (22), $u_{0} \in W^{1, \infty}(\mathbb{R})$ and $u$ be the solution of (21).

(i) If $u_{0}$ is a subsolution of $H\left(u_{x}\right)=0, x \in \mathbb{R}$ then

$$
\lim _{t \rightarrow+\infty} u(x, t)=\sup _{y \in \mathbb{R}} u_{0}(y)=: \varphi_{M}, \text { uniformly for } x \text { in compact sets of } \mathbb{R} .
$$

(ii) If $u_{0}$ is a supersolution of $H\left(u_{x}\right)=0, x \in \mathbb{R}$ then

$$
\lim _{t \rightarrow+\infty} u(x, t)=\inf _{y \in \mathbb{R}} u_{0}(y)=: \varphi_{m}, \text { uniformly for } x \text { in compact sets of } \mathbb{R} .
$$

Proof First notice that under the above assumptions, the problem (21) admits a unique bounded Lipschitz continuous solution. If $u_{0}$ is a subsolution of $H\left(u_{x}\right)=0$, then one knows that $u(x, t)$ is non decreasing in time and converges as $t$ goes to infinity, uniformly for $x$ in compact sets of $\mathbb{R}$, towards the minimal solution $\varphi$ of $H\left(u_{x}\right)=0$ which satisfies $\varphi(x) \geq u_{0}(x), x \in \mathbb{R}$. But as (22) holds, Proposition 1.3 applies and therefore $\varphi$ is necessarily a constant. Thus $\varphi(x) \equiv \varphi_{M}=\sup _{y \in \mathbb{R}} u_{0}(y)$. The second part (ii) follows in similar way. For results concerning long time behaviour of solutions of Hamilton-Jacobi equations, one can for example refer to the papers [1] and [6], see also [2].

Remark 2 Notice that the hamiltonian of the above example can be quite general with no particular property of convexity or superlinearity type.

Now let us turn to an example with a periodic source term. Consider the initial value problem

$$
\begin{cases}\partial_{t} u+\sqrt{1+\left(\partial_{x} u\right)^{2}}-1=\cos t, & (x, t) \in \mathbb{R} \times] 0,+\infty[, \\ u(x, 0)=u_{0}(x), & x \in \mathbb{R} .\end{cases}
$$

Proposition 2.2 For any $u_{0} \in W^{1, \infty}(\mathbb{R})$ the solution of (23) verifies

$$
\lim _{t \rightarrow+\infty}\{u(x, t)-\sin t\}=\inf _{y \in \mathbb{R}} u_{0}(y) \text {, uniformly for } x \text { in compact sets of } \mathbb{R} \text {. }
$$

Proof We will again use known results on the existence of time periodic solutions and on their asymptotic behaviour. We refer to [2] for example. First we know that (23) admits $2 \pi$ periodic solutions. This comes from the solvability of $\sqrt{1+\left(u^{\prime}\right)^{2}}-1=(2 \pi)^{-1} \int_{0}^{2 \pi} \cos (t) \mathrm{d} t=0, x \in \mathbb{R}$ (constants are solutions). Then observe that any $u_{0} \in W^{1, \infty}(\mathbb{R})$ is a supersolution of $\sqrt{1+\left(u^{\prime}\right)^{2}}-1=0$ so that

$$
\lim _{t \rightarrow+\infty}\{u(x, t)-\sin t\}=\psi(x) \text {, uniformly for } x \text { in compact sets of } \mathbb{R},
$$

where $\psi$ is the maximal solution of $\sqrt{1+\left(u^{\prime}\right)^{2}}-1=0, x \in \mathbb{R}$ such that $\psi(x) \leq u_{0}(x), x \in \mathbb{R}$. Now as $0 \notin \overline{I_{H}-\{0\}}=\emptyset$, by Proposition 1.3 we deduce that $\psi$ can only be a constant. The maximal constant $\psi$ verifying $\psi(x) \leq u_{0}(x), x \in \mathbb{R}$ is necessarily given by the infimum of $\left\{u_{0}(y): y \in \mathbb{R}\right\}$. 
Remark 3 In fact in the above example we are just recovering known results for convex hamiltonians, see [5] pp. 251. Indeed, as in this case $H$ is convex in $p$, one can obtain explicitly the solution via the Hopf-Lax formula applied to the equation satisfied by $v(x, t)=u(x, t)-\sin t$.

\section{Acknowledgement}

The authors are grateful to Prof. G. Barles and Prof. P. Cardaliaguet for helpful remarks and advices. We would like to thank Prof. P. Cardaliaguet for pointing out to us the main lines for the proof of Proposition 1.1 .

\section{References}

[1] G. Barles and P. E. Souganidis, On the large time behavior of solutions of Hamilton-Jacobi equations, SIAM J. Math. Anal. 31(2001), 925-939.

[2] M. Bostan and G. Namah, Time periodic viscosity solutions of Hamilton-Jacobi equations, Commun. Pure Appl. Anal. 6(2007), 389-410.

[3] P. Cannarsa and C. Sinestrari, Semiconcave functions, Hamilton-Jacobi equations and optimal control, Birkhäuser, Boston, 2004.

[4] L. C. Evans, Partial Differential Equations, American Mathematical Society, Providence, Rhode Island, 1998.

[5] P.-L. Lions, Generalized solutions of Hamilton-Jacobi Equations, Research Notes in Mathematics, Pitman, 1982.

[6] J.-M. Roquejoffre, Convergence to steady states or periodic solutions in a class of Hamilton-Jacobi equations, J. Math. Pures Appl. 80(2001), 85-104. 